\title{
SELECTION IN TWO ENVIRONMENTS
}

J. W. JAMES

Department of Animal Husbandry, University of Queensland, Brisbane

\section{INTRODUCTION}

Received 27.i.6r

A FREQUENTLY recurring problem of animal breeders is that of carrying out selection in the presence of interactions between genotype and environment. At one time it was widely believed that the only solution was to select different strains in every environment, so as to evolve one strain adapted to each (see, for example, Wright, 1939). However, Hammond (1947) suggested that animals should be selected for production "under environmental conditions which favour its fullest expression ", provided that the interactions were not of very great magnitude.

It was later shown by Falconer (1952) that there is no general solution to the problem, and that each case must be treated individually. Falconer treated the problem quantitatively by treating performances in two environments as genetically correlated characters, and using techniques already devised to deal with such characters. This concept has proved of great value in the interpretation of many experimental studies since reported and reviewed by McBride (1958).

It is the object of this paper to develop further some statistical aspects of the problem by extending Falconer's (1952) treatment to the case where genetic gain in both environments is desired, though the two environments may differ in importance. Such situations will arise particularly when only one or a few highly improved strains are to be used in a wide range of environments.

\section{DERIVATIONS}

Briefly, the approach initiated by Falconer is as follows. If $p_{A}$ and $p_{B}$ are the potential phenotypes of an individual if placed in environments $A$ and $B$ respectively, we write

$$
\begin{aligned}
& p_{A}=g_{A}+e_{A} \\
& p_{B}=g_{B}+e_{B}
\end{aligned}
$$

where $g$ is the breeding value and $e$ the environmental effect. We then impose the restriction that the covariances of all terms on the right hand sides of these equations are zero, except that

$$
E\left(g_{A} g_{B}\right)=r_{G} \sigma_{\sigma_{A}} \sigma_{g_{B}}
$$

where the $\sigma_{g}$ 's are the genetic standard deviations. It is then readily 
seen that if $\Delta_{A} g_{A}$ and $\Delta_{B} g_{A}$ are the respective gains in $g_{A}$ obtained by selection for $p_{A}$ and $p_{B}$, then we have

$$
\begin{aligned}
& \Delta_{A} g_{A}=\tau h_{A} \sigma_{g_{A}} \\
& \Delta_{B} g_{A}=\tau r_{G} h_{B} \sigma_{g_{A}}
\end{aligned}
$$

where $\tau$ is the selection differential in standard deviations and $h_{A}^{2}, h_{B}^{2}$ are the heritabilities in the two environments. It is then clear that if $\Delta_{B} g_{A}>\Delta_{A} g_{A}$ we must have $r_{G} h_{B}>h_{A}$. There are similar results for $\Delta_{B} g_{B}$ and $\Delta_{A} g_{B}$, the meanings of which terms are obvious.

The question now arises as to how selection should be made if performance in neither environment is irrelevant. This is simply dealt with in the normal manner by letting a gain in $g_{A}$ have $w$ times the value of an equal gain in $g_{B}$. If then $\Delta_{A} g$ is the overall genetic gain by selection for $p_{A}$ we have at once

and

$$
\begin{aligned}
& \Delta_{A} g=w \Delta_{A} g_{A}+\Delta_{A} g_{B} \\
& \Delta_{B} g=w \Delta_{B} g_{A}+\Delta_{B} g_{B} .
\end{aligned}
$$

Writing $\sigma_{g_{A}} / \sigma_{g_{B}}$ as $H$ for convenience, we have from above

$$
\frac{\Delta_{B} g}{\Delta_{A} g}=\frac{h_{B}}{h_{A}}\left[\frac{\mathrm{I}+w H r_{G}}{w H+r_{G}}\right] .
$$

This is conveniently rewritten as

$$
\frac{\Delta_{B} g}{\Delta_{A} g}=\frac{h_{B}}{h_{A}}\left[r_{G}+\frac{\mathrm{I}-r_{G}^{2}}{r_{G}+w H}\right]
$$

for comparison with $\Delta_{B} g_{A} / \Delta_{A} g_{A}$. It can be seen that Falconer's result corresponds to the special case when $w=0$. A point of interest here is that when $w H=\mathrm{I}$ the equation reduces to

$$
\frac{\Delta_{B} g}{\Delta_{A} g}=\frac{h_{B}}{h_{A}}
$$

and in this case the extent of the interaction between genotype and environment is irrelevant.

So far it has been assumed that only one environment is to be used, but it is possible to select two strains, one in each environment, and denoting the overall gain from this procedure as $\Delta g$, we have

$$
\Delta g=w \Delta_{A} g_{A}+\Delta_{B} g_{B} \text {. }
$$

From this it is easily shown that

$$
\frac{\Delta_{A} g}{\Delta g}=\mathrm{I}-\frac{\mathrm{I}-r_{G} \frac{h_{A}}{h_{B}}}{\mathrm{I}+w H_{\frac{h_{A}}{h_{B}}}^{h}}
$$

assuming that the intensity of selection is the same in both populations.

It then follows that $\Delta_{A} g>\Delta g$ if and only if $r_{G} h_{A}>h_{B}$, which result is of course as expected. 
There is yet another approach, which gives rise to a different problem. The breeder may wish to test one strain in each of two environments so that it is adapted to both, and select on an index combining performances in both environments. An individual cannot usually be itself tested in both environments, but such procedures as progeny testing provide a suitable method. For instance, in progeny testing to improve egg production in the domestic fowl, groups of daughters from each tested cockerel may be divided, some being tested on farms with an endemic disease, others on farms where this disease is absent. Then the two " phenotypes" of a cockerel are the means of his progeny groups.

The selection index is taken to be of the form

$$
I=k p_{A}+p_{B}
$$

and the problem is to find the value of $k$ which maximises overall genetic gain as previously defined. The general method of solution is well known, e.g. Smith (1936) and Hazel (1943). The special conditions associated with the present problem provide a particular solution, and this turns out to be

$$
k=\frac{w h_{A}^{2}}{h_{B}^{2}}\left[\frac{\left(\mathrm{I}-r_{G}^{2}\right)+r_{G}\left(\mathrm{I}-h_{B}^{2}\right)\left(r_{G}+\frac{\mathrm{I}}{w H}\right)}{\left(\mathrm{I}-r_{G}^{2}\right)+r_{G}\left(\mathrm{I}-h_{A}^{2}\right)\left(r_{G}+w H\right)}\right] .
$$

It should be remembered that in the application to a case such as outlined above the parameters in this equation are those associated with progeny groups. These are well-known (Lerner, I958) and need not be discussed here.

The expression for $k$ is rather formidable and some special cases are now given in illustration. When $r_{G}=0$ we have $k=w \frac{h_{A}^{2}}{h_{B}^{2}}$, the general result for uncorrelated characters. When $r_{G}=\mathrm{I}$ we have $k=$ $H \sigma_{e_{B}}^{2} / \sigma_{e_{A}}^{2}$, and when $w H=1$ we have

$$
k=w \frac{h_{A}^{2}}{h_{B}^{2}}\left[\frac{\mathrm{I}-r_{G} h_{B}^{2}}{\mathrm{I}-r_{G} h_{A}^{2}}\right] .
$$

This last value will usually be very close to that for uncorrelated characters.

When $w=o$ we can find that

$$
k=\frac{h_{A}}{H h_{B}} \frac{r_{G} h_{A}}{h_{B}} \cdot \frac{\mathrm{I}-h_{B}^{2}}{\mathrm{I}-\left(r_{G} h_{A}\right)^{2}} .
$$

In the case where $\Delta_{A} g_{B}=\Delta_{B} g_{B}$ we have $r_{G} h_{A}=h_{B}$ and the equation becomes

$$
k=\frac{h_{A}}{H h_{B}}=\frac{\sigma_{p_{A}}}{\sigma_{p_{B}}} .
$$


If index selection is to be preferred its greater operational difficulty must be repaid by considerable increases in genetic gain. If then $\Delta_{I} g$ is the overall gain by index selection, we must compare this with $\Delta_{B} g$ and $\Delta g$ as previously defined. The general expression for $\Delta_{I} g$ is extremely complicated and the general comparisons have not been made. However, when $w H=$ I the comparisons become much simpler and we may use this case as a guide to the general situation. Comparing then $\Delta_{I} g$ with $\Delta_{B} g$, it turns out that we have

$$
\frac{\Delta_{B} g}{\Delta_{I} g}=h_{B} \sqrt{\frac{\mathrm{I}-r_{G}^{2} h_{A}^{2} h_{B}^{2}}{h_{A}^{2}+h_{B}^{2}-2 r_{G} h_{A}^{2} h_{B}^{2}}} .
$$

Now, assuming that $h_{B}^{2}-h_{A}^{2}$ is small compared with $h_{B}^{2}$ we can write this approximately as

$$
\frac{\Delta_{B} g}{\Delta_{I} g}=\sqrt{\frac{\mathrm{I}+r_{G} h_{B}^{2}}{2}} .
$$

It is thus unlikely that $\Delta_{B} g$ will be more than about 80 per cent. as great as $\Delta_{I} g$ so that index selection has a very considerable advantage.

It may be noted also that this is the approximate result obtained for $w=0$ and $r_{G} h_{A}=h_{B}$ approximately.

Once more taking $w H=\mathrm{I}$ we have

$$
\frac{\Delta_{I} g}{\Delta g}=\frac{\mathrm{I}+r_{G}}{h_{A}+h_{B}} \sqrt{\frac{h_{A}^{2}+h_{B}^{2}-2 r_{G} h_{A}^{2} h_{B}^{2}}{\mathrm{I}-r_{G}^{2} h_{A}^{2} h_{B}^{2}}}
$$

and using the same approximation as before we find that this reduces to

$$
\frac{\Delta_{I G}}{\Delta_{G}}=\sqrt{\frac{\left(\mathrm{I}+r_{G}\right)^{2}}{2\left(H r_{G} h_{B}^{2}\right)}} .
$$

From this it follows that index selection will usually be more efficient

\begin{tabular}{|c|c|c|c|c|c|}
\hline \multirow{2}{*}{$r_{G}$} & \multicolumn{5}{|c|}{$w H$} \\
\hline & $\frac{1}{2}$ & $2 / 3$ & I & $1 \frac{1}{2}$ & 2 \\
\hline $\begin{array}{l}\cdot 0 \\
0.25 \\
0.50 \\
0.75 \\
1.0\end{array}$ & $\begin{array}{l}1 \cdot 114 \\
1 \cdot 326 \\
1 \cdot 570 \\
1 \cdot 915 \\
2 \cdot 533\end{array}$ & $\begin{array}{l}I \cdot 114 \\
I \cdot 238 \\
I \cdot 379 \\
I \cdot 572 \\
I \cdot 900\end{array}$ & $\begin{array}{l}I \cdot I 144 \\
I \cdot I 33 \\
I \cdot 160 \\
I \cdot 200 \\
I \cdot 267\end{array}$ & $\begin{array}{l}\mathrm{I} \cdot \mathrm{I} 14 \\
\mathrm{I} \cdot 039 \\
0.987 \\
0.917 \\
0.844\end{array}$ & $\begin{array}{l}I \cdot I 14 \\
0.973 \\
0.861 \\
0.754 \\
0.633\end{array}$ \\
\hline
\end{tabular}
provided that $r_{G}>0 \cdot 7$ approximately.

TABLE I

Values of $\frac{\mathrm{k}}{\mathrm{w}}$ for specified values of $\mathrm{r}_{\mathrm{G}}$ and $\mathrm{wH}$ for $\mathrm{h}_{\mathbf{A}}^{2}=0.57, \mathrm{~h}_{\mathbf{B}}^{2}=0.5 \mathrm{I}$

This would apply with progeny groups of size 20 with individual heritabilities of 0.25 and $0 \cdot 20$. 
As a further illustration of the equation for $k$, tables $\mathrm{I}$ and 2 give the values of $k$ for some particular situations. In these tables it can be seen that over the range of $r_{G}$ from zero to one $k$ changes approximately linearly. Since it has been shown by Robertson (1959) that sampling errors in the estimation of $r_{G}$ are likely to be large, and all other parameters are also subject to errors of estimation, there may be little value

TABLE 2

Values of $\frac{\mathrm{k}}{\mathrm{w}}$ for specified $\mathrm{r}_{\mathrm{G}}$ and $\mathrm{wH}$ with $\mathrm{h}_{\mathrm{A}}^{2}=0.25, \mathrm{~h}_{\mathrm{B}}^{2}=0.20$

\begin{tabular}{|c|c|c|c|c|c|}
\hline \multirow{2}{*}{$r_{G}$} & \multicolumn{5}{|c|}{$w H$} \\
\hline & $\frac{1}{2}$ & $2 / 3$ & 1 & $3 / 2$ & 2 \\
\hline $\begin{array}{l}0 \cdot 0 \\
0 \cdot 25 \\
0 \cdot 5 \\
0 \cdot 75 \\
1 \cdot 0\end{array}$ & $\begin{array}{l}1 \cdot 25 \\
1 \cdot 61 \\
1 \cdot 94 \\
2 \cdot 29 \\
2 \cdot 67\end{array}$ & $\begin{array}{l}\mathbf{I} \cdot 25 \\
\mathbf{I} \cdot 45 \\
\mathrm{I} \cdot 63 \\
\mathbf{1} \cdot 8 \mathrm{1} \\
\mathbf{2} \cdot 00\end{array}$ & $\begin{array}{l}1 \cdot 25 \\
1 \cdot 27 \\
1 \cdot 29 \\
1 \cdot 31 \\
1 \cdot 33\end{array}$ & $\begin{array}{l}\mathrm{I} \cdot 25 \\
\mathrm{I} \cdot 1 \mathrm{I} \\
\mathrm{I} \cdot 01 \\
0 \cdot 94 \\
0.89\end{array}$ & $\begin{array}{l}1 \cdot 25 \\
1 \cdot 00 \\
0.85 \\
0.75 \\
0.67\end{array}$ \\
\hline
\end{tabular}

This would apply for progeny groups of size 20 and individual heritabilities of 0.066 and $0 \cdot 050$.

in extreme accuracy in the calculation of $k$ from these parameter estimates. It is therefore suggested that a satisfactory estimate of $k$ may be obtained by linear interpolation between $w \frac{h_{A}^{2}}{h_{B}^{2}}$ and $H \frac{\sigma_{e B}^{2}}{\sigma_{e A}^{2}}$ at the estimated value of $r_{G}$.

It may be worth pointing out that these results apply to correlated characters in general, provided these correlations are genetic only. This may be compared with the method of Rendel (1954) to deal with characters environmentally correlated.

Exactly the same method may be applied to selection in more than two environments, but there seem to be no useful simplifications such as arise in the case of two environments. It is simple to write out the equations requiring solution.

\section{DISCUSSION}

In recent years there has been a tendency for the means of genetic improvement of domestic animals to be concentrated in the hands of a few large breeders. This has been particularly marked in the poultry industry. Under such conditions, the ability of a breeder's stock to produce well over a wide range of environments becomes extremely important, and what has here been called overall genetic gain will be the object of selection programmes.

The simplest approach is of course to ignore genotype-environment interactions, and if this is done selection should be carried out in the 
environment giving greater overall gain. For two environments this has been shown to depend on $h_{A}^{2}, h_{B}^{2}, H, w$ and $r_{G}$. Table 3 gives values of $h_{B}^{2} / h_{A}^{2}$ which must be exceeded for given values of $r_{a}$ and $w H$ if $p_{B}$ is to be selected for in preference to $p_{A}$.

Over what seems likely to be the most important part of the range $\left[2 / 3<w H<3 / 2: 0 \cdot 7<r_{G}<\right.$ I] it can be seen that if one heritability is about $I_{1} I_{5}$ times as large as the other, this will be a sufficient increase to determine which environment should be used. For instance, if $w H=2 / 3, r_{G}=0.7, h_{A}^{2}=0.25$, then if $h_{B}^{2}$ is less than 0.217 selection should be for $p_{A}$. However, the differences in gains are small unless $h_{B}^{2}$ is much less than 0.217 , and in practice the final decision would

TABLE 3

Values of $\frac{\mathrm{h}_{\mathrm{B}}^{2}}{\mathrm{~h}_{\mathrm{A}}^{2}}$ for which $\Delta_{\mathrm{B}} \mathrm{g}=\Delta_{\mathrm{A}} \mathrm{g}$ if $\frac{\mathrm{h}_{\mathrm{B}}^{2}}{\mathrm{~h}_{\mathrm{A}}^{2}}>$ tabulated value, $\Delta_{\mathrm{B}} \mathrm{g}>\Delta_{\mathrm{A}} \mathrm{g}$

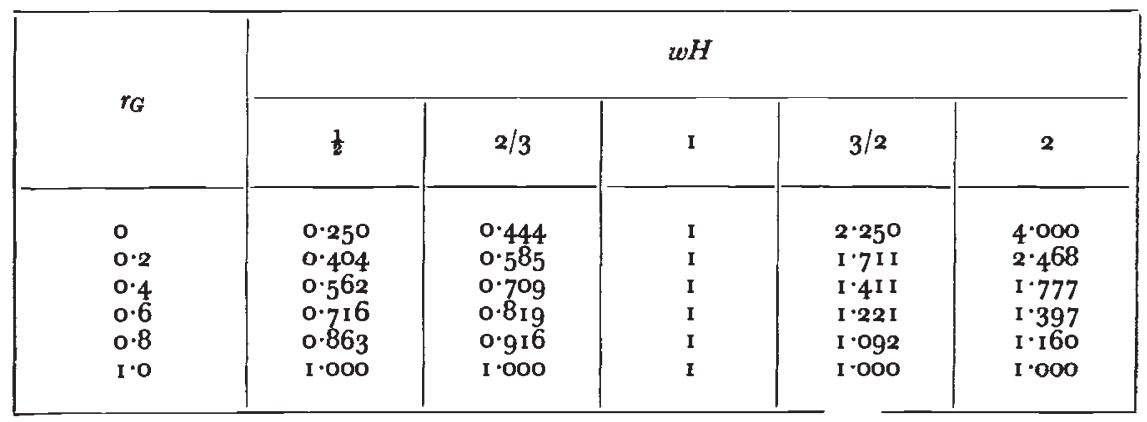

usually be based on the operational convenience of the two environments.

It may often be desirable to avoid difficulties involved in the maintenance, testing and selection of two separate strains, especially when there are chances that the purchaser will use one strain in an environment different from that for which it was developed. Under these conditions it would seem best to develop a strain adapted to both environments, and the results given above suggest that a selection index will be the most efficient method of developing such a strain. However, the comparison given above of overall gains from index selection with those from separate selection will usually be biased in favour of the index. This follows from the assumptions that selection intensity is the same in both strains, and that population size is the same for each strain. These objections appear to be important only if $w$ deviates considerably from one, so the bias may not be very great in many cases.

It might appear that use of the index would necessitate the doubling of testing facilities, but this is by no means always correct. For instance, if poultry are tested on outside farms, it involves a splitting of these 
farms into two groups and extra work in data processing, which does not double the operational problems.

One interesting result of the experiments of Falconer and Latyszewski (1952) and Fowler and Ensminger (1957) has been that selection in "optimal" (A) conditions has been less efficient than selection in "sub-optimal" (B) conditions. In such situations it seems in general that $h_{A}^{2}>h_{B}^{2}$ and $H<\mathrm{I}$. We here consider a hypothetical case of this type. We take $h_{A}^{2}=0.25, h_{B}^{2}=0.20, H=0.7, w=\mathrm{I}, r_{G}=0.4$. Then we have $\frac{\Delta_{B} g}{\Delta_{A} g}=\mathrm{I} \cdot 04$. Substitution of these values in the index equation gives the result $k=\mathrm{I} \cdot 523$. The use of the suggested linear interpolation would yield $k=\mathrm{I} \cdot 5 \mathrm{I} 2$. It is noteworthy that in this example the phenotype which is less efficient alone is given the greater weight in the index. Such a result is by no means intuitively obvious.

When the actual comparison is made we find $\frac{\Delta_{B} g}{\Delta_{I} g}=0 \cdot 75^{26}$. Using the approximate formula given earlier we would obtain $\frac{\Delta_{B} g}{\Delta_{I} g}=0 \cdot 734$. This example shows how the suggested approximations work in a particular case.

It should be noted that the present formulation differs from Falconer's (1952) in two respects. His path diagram may be written as

$$
\begin{aligned}
& p_{A}=g_{A}+e_{A}+t_{A} \\
& p_{B}=g_{B}+e_{B}+t_{B}
\end{aligned}
$$

where the $t$ 's are effects associated with specific environments, while the $e$ 's are random variables within these environments. In the present model these have been amalgamated. A more important difference is the present assumption that $E\left(e_{A} e_{B}\right)=0$ which is not made by Falconer. This does not affect the results except in the derivation of the index. However, in the situation discussed, where "phenotypes" are means of related groups, this assumption is simply that commonly made of no environmental correlations between relatives. For completeness we may include the equation for $k$ when $r_{P} \neq r_{G} h_{A} h_{B}$. It is

$$
k=w \frac{h_{A}^{2}}{h_{B}^{2}}\left[\frac{\left(\mathrm{I}+\frac{r_{G}}{w H}\right)-\left(\frac{\mathrm{I}}{w H}+r_{G}\right) r_{P} \frac{h_{B}}{h_{A}}}{\left(\mathrm{I}+w H r_{G}\right)-\left(w H+r_{G}\right) r_{P} \frac{h_{B}}{h_{A}}}\right] .
$$

which clearly reduces to that given earlier when $E\left(e_{A} e_{B}\right)=0$.

It is very doubtful whether the simple model presented here would give accurate predictions over an extended period. However, it seems probable that the index method may be of considerable value over a few generations, after which the selection procedure would need to be revised in the light of results. 


\section{SUMMARY}

I. The "overall gain "through selection is defined when animals are to be used in two environments.

2. Three selection procedures are considered:

(i) Selection in one environment ;

(ii) Separate selection in both environments of two strains ;

(iii) Selection on an index combining performance in both environments.

3. Expected overall gain is worked out for each procedure, and the optimum index is derived.

4. Comparison of gains is made to give a basis for choosing the form selection should take. Over a range of conditions index selection is most efficient, producing greater overall gain than even separate selection.

5. The implications of these results are discussed.

Acknowledgments.-I wish to thank the Rural Credits Development Fund of the Reserve Bank of Australia for a Fellowship during the tenure of which this work was done. I wish also to thank $\mathrm{Dr}$ G. McBride for his helpful comments.

\section{REFERENCES}

FALCONER, D. S. 1952. The problem of environment and selection. Amer. Nat., $86,293-298$.

FALCONER, D. S., AND LATYSZEWSKI, M. 1952. The environment in relation to selection for size in mice. 7. Genet., 5I, 67-80.

FOWLER, S. H., AND ENSMINGER, M. E. 1957. Full vs. limited feeding as related to the improvement of animals for meat production through breeding. F. Anim. Sci., 16, 1049.

HAMMOND, J. 1947. Animal breeding in relation to nutrition and environmental conditions. Biol. Rev., 22, 195-213.

HAZEL, L. N. 1943. The genetic basis for constructing selection indexes. Genetics, $28,476-490$.

LERNER, I. M. 1958. The genetic basis of selection. John Wiley and Sons, New York.

MCBRIDE, G. 1958. The environment and animal breeding problems. Anim. Breed. Abstr., 26, 340-358.

RENDEL, J. M. 1954. The use of regression to increase heritability. Aust. F. Biol. Sci., $7,368-378$.

ROBERTSON, A. I959. The sampling variance of the genetic correlation coefficient. Biometrics, $15,469-485$.

sмiтн, н. F. 1936. A discriminant function for plant selection. Ann. Eugen. (Lond.), $7,240-250$.

WRIGHT, s. 1939. Genetic principles governing the rate of progress of livestock breeding. Proc. Amer. Soc. Anim. Prod. 32nd Ann. Meet., 18-26. 